\title{
A CFD STUDY ON TWO-PHASE FROZEN FLOW OF AIR/WATER THROUGH A SAFETY RELIEF VALVE
}

\author{
Moftah Alshaikh ${ }^{a}$, William Dempster ${ }^{b}$ \\ ${ }^{a}$ Department of Mechanical and Aerospace Engineering, University of Strathclyde , \\ Montrose St ,Glasgow, G1 1 XJ. Email: moftah-s--moftah-alshaikh@strath.ac.uk \\ ${ }^{b}$ Department of Mechanical and Aerospace Engineering, University of Strathclyde , \\ Montrose St ,Glasgow, G1 1 XJ. Email: william.dempster@strath.ac.uk
}

\begin{abstract}
The air-water two phase critical flows through a safety relief valve commonly used in the refrigeration industry is examined with particular emphasis on the prediction of the critical mass flowrates using CFD based approaches. The expansion of the gas through the valve and the associated acceleration is coupled to the liquid phase and results in changes to the velocity slip with the possibility of influencing the choking conditions and the magnitude of the critical mass flows. These conditions are poorly reported in the literature for safety valves. This paper presents a study where the ability of established two phase multi-dimensional modelling approaches to predict such conditions are investigated. Comparison with the simplified mixture model will show that this model tends to underestimate mass flowrates for medium to high liquid mass fraction. However, the two fluid model can adequately account for the thermal and mechanical non equilibrium for these complex flow conditions with the use of simplified droplet sizing rules.
\end{abstract}

Keywords: Two Phase Flow, Safety Valves, CFD, Experiments

Nomenclature

$\mathrm{A}_{i}$

$\mathrm{C}_{\mathrm{d}}$

$\mathrm{C}_{\mathrm{p}}$

$\mathrm{d}_{\mathrm{d}}$

$\mathrm{f}$

g

$\mathrm{h}_{\mathrm{q}}$

$\mathrm{k}$

$\mathrm{K}_{\mathrm{pq}}$

$\mathrm{Nu}_{\mathrm{d}}$

$\mathrm{P}$

$Q_{p q}$

$\mathrm{R}_{\mathrm{pq}}$

$\mathrm{Re}$

$\mathrm{T}$

V

$\vec{V}_{d r}$ phase interfacial area

drag coefficient

specific heat

droplet diameter

Friction factor

gravitational acceleration

phase enthalpy

thermal conductivity

momentum exchange

Nusselt number- droplet

pressure

Interphase heat transfer

phase interaction force

Reynold number

temperature

velocity

drift velocity due to

\section{Greek}

$\alpha \quad$ void fraction

$\mu \quad$ viscosity

$\mu_{\mathrm{t}} \quad$ turbulent viscosity

$\mu_{\mathrm{t}, \mathrm{q}} \quad$ phase turbulent viscosity

$\mu_{\mathrm{t}, \mathrm{m}} \quad$ mixture turbulent viscosity

$\tau_{\mathrm{d}} \quad$ droplet relaxation time

$\varepsilon \quad$ turbulent dissipation

$\kappa \quad$ turbulent kinetic energy

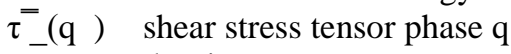

$\rho$ density

$\rho_{\mathrm{m}} \quad$ mixture density

$\sigma_{\mathrm{k}} \quad$ Prandtl number $-\mathrm{k}$

$\sigma_{\varepsilon} \quad$ Prandtl number $-\varepsilon$

$\lambda$ bulk viscosity

indices

p, q phase indicator 


\section{INTRODUCTION}

In a wide variety of engineering systems such as heat exchangers, power systems, fluid transport systems and chemical plant, safe operation is achieved by the use of safety relief valves to limit excessive pressure levels. The analysis of single phase flows and the determination of mass flows in safety valves is largely understood both experimentally and theoretically with CFD approaches having being successfully applied as shown by Dempster et.al, 2006, Schmidt and Egan, 2009 and Song et.al, 2014. However, the operation of safety valves under two-phase flow conditions is less well understood particularly when non equilibrium effects due to phase change occur. Here the rapid expansion in the valve often results in liquid flashing or droplet condensation depending on the upstream initial conditions. The resulting mass exchange controls the critical flow conditions which determine the valve flowrate. At present the most widely accepted methods of predicting the relieving capacity of valves under two-phase flow conditions are the algebraic based homogenous equilibrium model (HEM) developed by Leung, 1986 or the homogenous non-equilibrium model of Diener and Schmidt (HNEM-DS), (Schmidt and Egan, 2009) proposed in ISO-4126-10, (ISO -4126, 2010). Most of the international standard design methods have led to either oversizing or undersizing of conventional safety relief valves. Although the continuous improvement of the two phase physical models and numerical methods makes simulations of the very complex flows in safety valves now possible, there are only a limited number of publications showing their capability. Dempster and Elmayyah (2013), predicted the critical flow condition through a conventional safety relief valve using a simplified CFD mixture model for the multiphase air/water flows. The mixture model was examined by using the commercial code FLUENT 6.3.2. This mixture model assumes that both phases are at mechanical and thermal equilibrium conditions. The study showed good agreement between the CFD results and the experiments up to $40 \%$ liquid mass fraction. Interestingly the HEM method of Darby, 2004 and the CFD results also matched these conditions. However, an increasing discrepancy of the order of $15 \%$ under prediction was evident for larger liquid mass fractions from 40 to $90 \%$. For the larger liquid mass fractions it was surmised that the effects of non-equilibrium increased due to the increase in liquid inertia, leading to an increase in velocity slip and an increase in liquid thermal inertia resulting in poorer mass flow predictions. This paper investigates these issues further by examining the use of an Euler-Euler two fluid model to account for the non-equilibrium effects and investigate the capability of established closure models to predict the valve mass flows.

\section{Safety Valve Details}

A conventional spring loaded safety relief valve commonly used in refrigeration systems was investigated in this study. Figure 1 depicts a cross section of $1 / 4$ " inlet bore size of the safety relief valve which comprises a movable piston, loaded by a spring to the required relief pressure. The spring load is retained and applied by an adjustable gland. The valve establishes a seal through a piston sealing disk inserted into the piston which sits on the valve seat machined into the valve body, to maintain the system pressure. The operation of the valve is mainly a dynamic process therefore exceeding the set pressure leads to the piston lifting to allow mass to discharge via an available flow area. The piston lift relies essentially on the increasing system pressure which is expected to be limited due to the greater flow discharged. The current work is simplified as quasi static conditions are postulated assuming that pressure waves can be ignored. Thus the flow in the valve is adequately represented by a series of steady state conditions. It is expected that this would be more appropriate for the closing operation of a safety relief valve than the opening phase. 


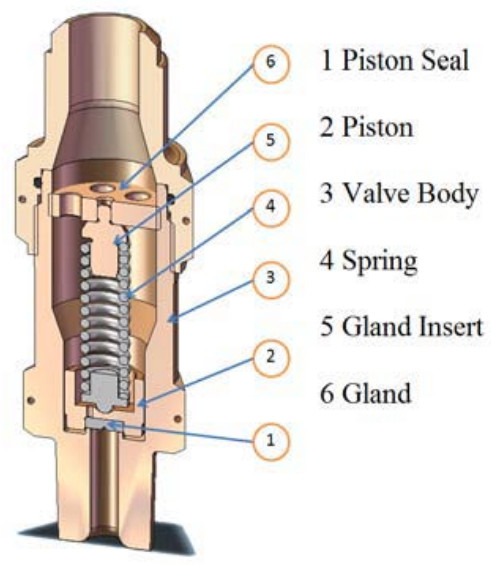

Fig.1: Safety Relief Valve

The flow path through the valve is complex and generally multi-dimensional distinguishing it from previous detailed modelling studies of critical flow where simple nozzles have been examined. In this paper the main focus of attention are the flows in the vicinity of the seat and piston since this is where the first occurrence of critical flow occurs and controls the valves mass flowrate. To achieve this, the flow undergoes a series of contractions and expansions in an annular space and can be modelled using two dimensional axi-symmetric assumptions.

\section{Experimental Set up and Procedures}

An experimental flow facility for valve testing under steady state flow conditions was developed to allow independent control of the valve piston position and upstream water injection at various operating upstream air pressure conditions. The rationale was to measure the air flowrates at constant upstream pressure for variations in injected water flowrate. Thus, for each test condition, determined by setting the piston lift, upstream pressure and liquid flowrate, the air mass flow becomes the uncontrolled quantity determined by the circumstances of the two phase interaction with the valve. The water is injected into the flowing air upstream of the valve using a spray nozzle allowing the liquid to mix prior to entering the valve. Downstream of the valve, a separator is used to separate the water and the air. The water collected in the separator also acts as a water supply for the water injection pump. The test rig, Figure 2, consists of a $100 \mathrm{~mm}$ (4 inch) diameter pipe connected to a compressed air system providing air in the pressure range 1-15 barg to the valve. The tested safety valve is connected to the pipe via a brass converging section to adapt to the valve entrance. A liquid injection nozzle is fitted into the converging section to inject the water. The injection nozzle located in the centre of the pipe produces a uniform full cone spray with a low spray angle of $30^{\circ}$. A PVC T junction with a $50 \mathrm{~mm}$ diameter side exit is connected to the valve to direct the two phase mixture to a separator. The $\mathrm{T}$ junction could be maintained close to atmospheric pressure. It has a pressure tapping fitted to measure the pressure at the valve outlet. The valve piston is attached to a $250 \mathrm{~mm}$ long $6 \mathrm{~mm}$ diameter rod which passes through the far end of the PVC tube end and is connected to a lead screw and traverse table allowing the piston position to be adjusted. The piston movement is in the range of $0-5 \mathrm{~mm}$ and was measured by a Mitutoyo digital dial indicator with sensitivity of $0.001 \mathrm{~mm}$. The water injection system consists of a positive displacement diaphragm water pump (Hydra Cell D/G-04 series) connected to the injection spray nozzle via a high pressure hose. The pump has a maximum flowrate of $11 \mathrm{l} / \mathrm{min}$ and will deliver the flow independently of the downstream pressure up to 100 bar. The pump is driven by an AC motor controlled by a speed controller, 
which allows adjustment of the water flow rate. Upstream of the injection nozzle, a turbine flow meter (Omega Engineering FTB 1411) is fitted to facilitate measurement of the water flow rate; it has a flow rate range of $0.4-10 \mathrm{l} / \mathrm{min}$ and has an accuracy of $+/-1 \%$ of the reading. A bladder accumulator (FlowGuard DS-20) is also connected to the pump outlet to damp the pulsating water flow rate from the pump. The air flowrate was measured using a Sierra Vortex mass flowmeter (Innova mass 240) and accurate to $<1 \%$ of reading. The upstream pressure and outlet pressure are measured by Bourdon pressure gauges. The range of upstream pressures (1-15 bar) and water flow rates $(1-10 \mathrm{~L} / \mathrm{min})$ give a working air flow rate from 0.015 to $0.12 \mathrm{~kg} / \mathrm{s}$ and a water mass fraction range from 0 to 0.9 .

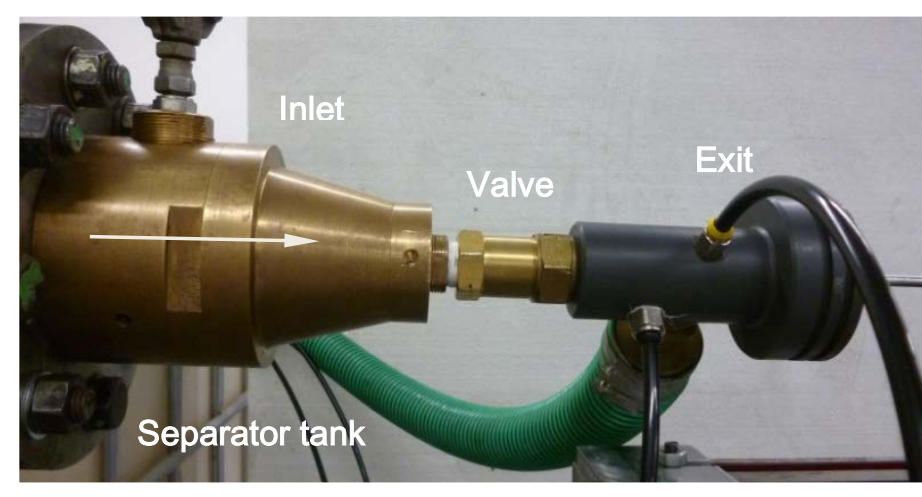

Liquid and air to separator/storage tank

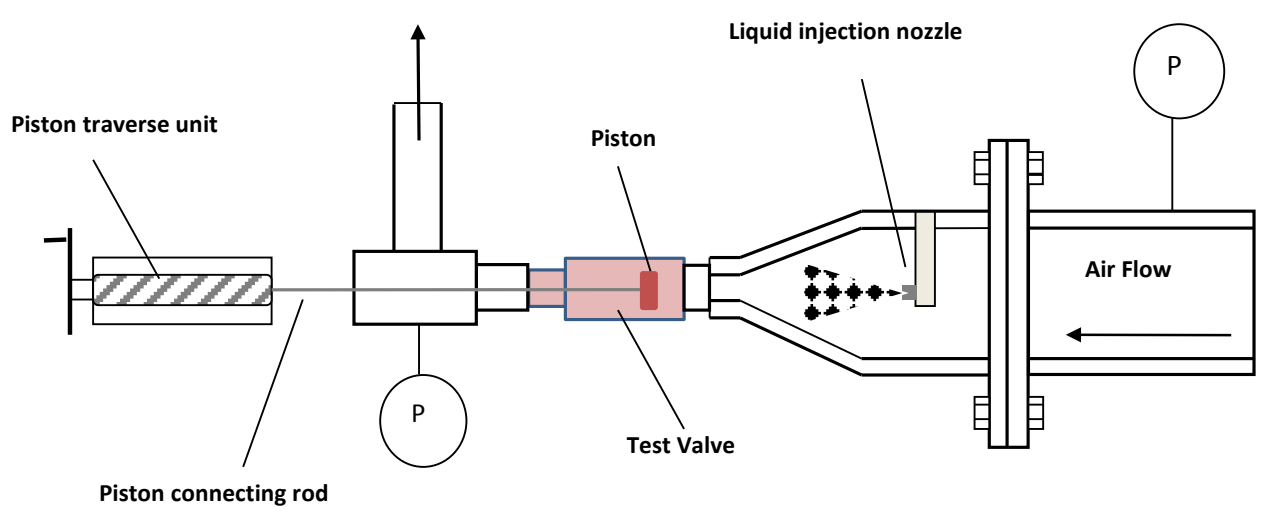

Fig. 2 Experimental Test Facility

Test data presented in this paper was taken at a pressure of 13.7 barg for a liquid flowrate of $0-10 \mathrm{~L} / \mathrm{min}$ and at a fixed piston lift position of $5 \mathrm{~mm}$, which corresponds to a fully open position of the valve. This allowed a 0-0.8 range of liquid mass fractions to be examined which influences the degree of mechanical and thermal non-equilibrium, ie velocity differences and the temperature changes during the expansion process. The temperature of the gas decreases during the expansion process as the fluid accelerates but will be restricted by the heat transfer from the liquid which changes less due its larger thermal inertia. Likewise the increasing amount of liquid requires to be accelerated by the air resulting in an interfacial resistance by the liquid. This effect will increase as the liquid mass flow increases. In this study the increased liquid flow acts as a means to introduce greater non equilibrium and takes the form of an independent variable. The effect of the air flow is thus investigated and becomes the dependent variable. 


\section{Modelling Approaches}

\subsection{Two phase models}

Two modelling approaches were investigated to address the two phase conditions (i) an Eulerian-Eulerian two fluid model which could address thermal and mechanical non equilibrium effects and (ii) a mixture model which describes the limiting homogenous flow conditions.

Conservation of mass

$$
\nabla \cdot\left(\begin{array}{ll}
\alpha_{q} \rho_{q} & \vec{V}_{q}
\end{array}\right)=0
$$

Compatibility condition

$$
\sum_{q=1}^{n} \alpha_{q}=1
$$

Momentum equation

$$
\nabla \cdot\left(\alpha_{q} \rho_{q} \vec{V}_{q} \vec{V}_{q}\right)=-\alpha_{q} \nabla P+\nabla \cdot \overline{\bar{\tau}}_{q}+\alpha_{q} \rho_{q} \vec{g}+\sum_{p=1}^{n} R_{p q}
$$

Auxiliary equations

$$
\begin{aligned}
\overline{\bar{\tau}}_{q} & =\alpha_{q}\left(\mu_{q}+\mu_{t, q}\right)\left(\nabla \vec{V}_{q}+\nabla \vec{V}_{q}^{t}\right)+\alpha_{q}\left(\lambda_{q}-\frac{2}{3}\left(\mu_{q}+\mu_{t, q}\right) \nabla \vec{V}_{q} I\right. \\
R_{p q} & =K_{p q}\left(\vec{V}_{p}-\vec{V}_{q}\right) \\
K_{p q} & =\frac{\rho_{p} f}{6 \tau_{d}} d_{d} A_{i} \quad A_{i}=\frac{6 \alpha_{p} \alpha_{p}}{d_{d}^{2}} \quad \tau_{d}=\frac{\rho_{p} d_{d}^{2}}{18 \mu_{q}} \quad f=\frac{C_{D} R e_{d}}{24} \quad \operatorname{Re}_{d}=\frac{\rho_{\mathrm{q}}\left|\vec{V}_{\mathrm{p}}-\vec{V}_{\mathrm{q}}\right| \mathrm{d}_{\mathrm{d}}}{\mu_{\mathrm{q}}} \\
C_{D} & =\left\{\begin{array}{cc}
\frac{24\left(1+0.15 R e_{d}^{0.687}\right)}{R e_{d}} & R e_{d} \leq 1000 \\
0.44 & R e_{d}>1000
\end{array}\right.
\end{aligned}
$$

Conservation of energy $\quad \nabla \cdot\left(\alpha_{q} \rho_{q} \vec{u}_{q} h_{q}\right)=\alpha_{q} \frac{\partial P_{q}}{\partial t}+\overline{\bar{\tau}}_{q}: \nabla \cdot \vec{u}_{q}-\nabla \cdot \vec{q}_{q}+\sum_{p=1}^{n}\left(Q_{p q}\right)$

Auxiliary equations $\quad Q_{p q}=h_{p q} A_{i}\left(T_{p}-T_{q}\right)$

$$
\begin{aligned}
h_{p q} & =\frac{k_{q} N u_{d}}{d_{d}} \quad A_{i}=\frac{6 \alpha_{\mathrm{p}} \alpha_{\mathrm{p}}}{\mathrm{d}_{\mathrm{d}}^{2}} \\
N u_{d} & =\left[2.0+0.6 \operatorname{Re}_{d}^{\frac{1}{2}} \operatorname{Pr}^{\frac{1}{3}}\right] \quad 0.1<R e_{d}<4000 \\
\operatorname{Pr} & =\frac{C_{p, q} \mu_{q}}{\mathrm{k}_{q}} . \quad \operatorname{Re}_{d}=\frac{\rho_{\mathrm{q}\left|\overrightarrow{\mathrm{v}}_{\mathrm{p}}-\overrightarrow{\mathrm{v}}_{\mathrm{q}}\right| \mathrm{d}_{\mathrm{d}}}}{\mu_{\mathrm{q}}}
\end{aligned}
$$

Turbulence model- $k$-e transport equations

$$
\begin{gathered}
\nabla \cdot\left(\rho_{m} \vec{v}_{m} \kappa\right)=\nabla \cdot\left\{\left(\mu_{m}+\frac{\mu_{t, m}}{\sigma_{k}}\right) \nabla \kappa\right\}+G_{\kappa, m}-\rho_{m} \varepsilon \\
\nabla \cdot\left(\rho_{m} \vec{v}_{m} \varepsilon\right)=\nabla \cdot\left\{\left(\mu_{m}+\frac{\mu_{t, m}}{\sigma_{\varepsilon}}\right) \nabla \varepsilon\right\}+\frac{\varepsilon}{\kappa}\left(C_{1 \varepsilon} G_{\kappa, m}-C_{2 \varepsilon} \rho_{m} \varepsilon\right) \\
\rho_{m}=\sum_{i=1}^{N} \alpha_{i} \rho_{i} \quad \mu_{m}=\sum_{i=1}^{N} \alpha_{i} \mu_{i} \quad \vec{v}_{m}=\frac{\sum_{i=1}^{N} \alpha_{i} \rho_{i} \vec{v}_{i}}{\sum_{i=1}^{N} \alpha_{i} \rho_{i}} \\
G_{\kappa, m}=\mu_{t, m}\left(\nabla \vec{v}_{m}+\left(\nabla \vec{v}_{m}\right)^{T}: \nabla \vec{v}_{m}\right. \\
\mu_{t, m}=\rho_{m} \cdot C_{\mu} \cdot \frac{\kappa^{2}}{\varepsilon} \quad \mu_{t, q}=\frac{\rho_{q}}{\rho_{m}} \mu_{t, m} \\
C_{\mu}=0.06 \quad C_{1 \varepsilon}=1.44, C_{2}=1.92, \sigma_{k}=1.0, \sigma_{\varepsilon}=1.3
\end{gathered}
$$


The mixture model follows Manninen and Taavassalo, (1996) and the equation system (1)-(4) describes the two fluid approach. The details of the governing equations of both models are described in FLUENT documentation (Fluent 2006). The equations are in volume averaged form. Interfacial drag, Equations (2a-2e) is determined by the drag coefficient model of Schiller and Naumann, (1935), the interfacial heat transfer is based on Ranz and Marshall's work, (1952). A simplified approach to two phase turbulence is chosen based on a k- $\varepsilon$ mixture turbulence approach (Fluent, 2006). The turbulence is coupled to the momentum equations through the shear stress tensor only.

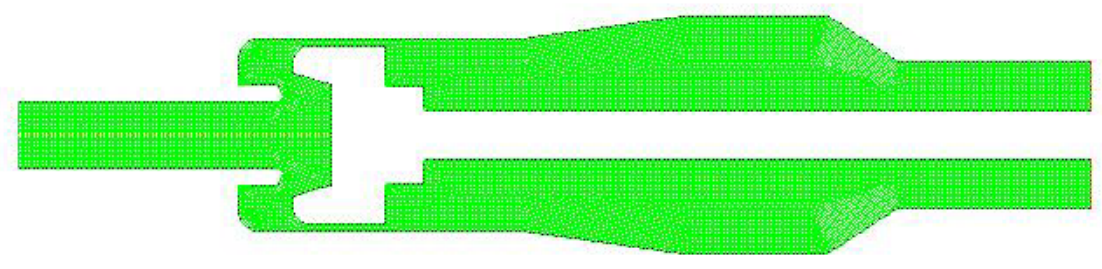

Fig.3: The computational model

A fully opened safety relief valve geometry with its adjusted gland is presented as a 2Daxisymmetric model with a structured mesh as shown on Fig.3. The mesh used for all calculations contains approximately 11350 elements and based on a quadrilateral cell type. On average the mesh density is 8 cells $/ \mathrm{mm}^{2}$ and has been found from Elmayyah (2010) to be sufficiently accurate. The mesh grid is generated based on the curvature advanced size function with a fine relevance centre as well as a fine span angle centre. The generated grid is also examined using the mesh metric methodology of skewness and found to be 0.69 as a maximum value in only 157 elements over the whole flow domain. An investigation into grid independence was carried out where the results from the standard mesh (11350 cells) were compared with those of a coarser mesh (7200 cells) and a mesh 5 times more dense with 66424 cells. The results are shown on Figure 4 and indicate that the 11350 cell mesh is grid independent as far as the mass flowrate predictions are concerned. The 11350 grid also ensured grid independence for other global parameters, such as piston force, (relevant for a parallel study on valve operation) and local flow parameters such as velocity, pressure and observed compressible shock structures. This was less assured for the 7200 cell grid as can be inferred from Fig. 4.

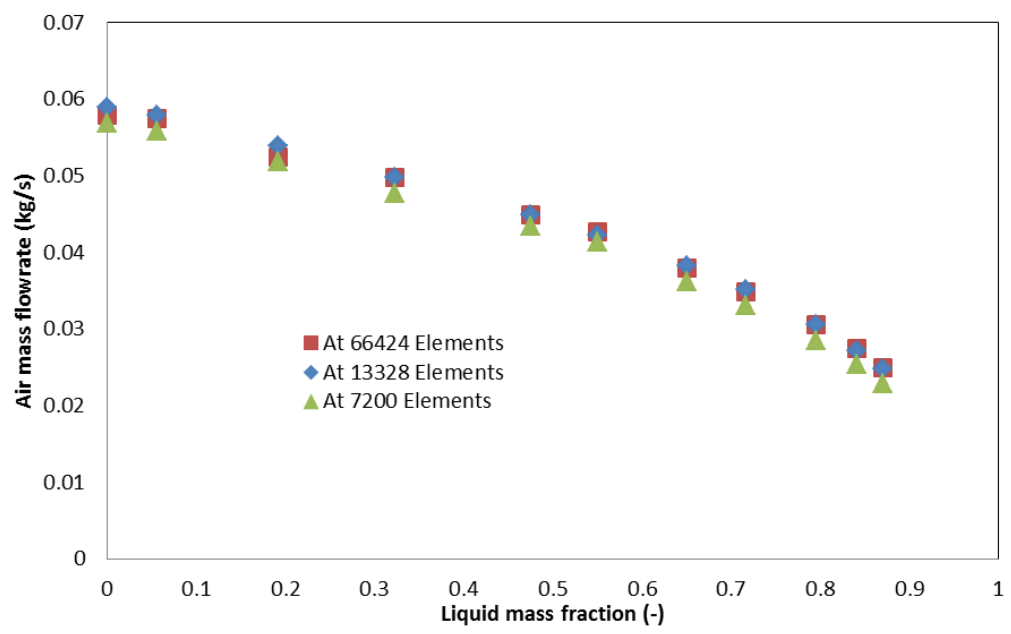

Fig. 4 Mesh independence study 


\subsection{Boundary conditions and solution strategy}

There are three main zones; the inlet, specified as a pressure-inlet, the outlet specified as a pressure-outlet and the interior-fluid represents the whole domain as an air/water mixture. Other zones are considered as walls. At the inlet-pressure boundary condition, the total pressure of the mixture is specified as $13.7 \mathrm{barg}$. The gas and liquid temperatures are set at the corresponding experimental values and are generally close to $288 \mathrm{~K}$. The liquid mass fraction has different values (from 0 to 0.8). As the mixture turbulence multiphase model is employed initial guess values of $\kappa$ and $\epsilon$ are set to be 0.1 and 0.2 , respectively. The pressure-outlet boundary is set at atmospheric pressure. Also, initial values of $\kappa$ and $\epsilon$ and the volume fraction of the water are to be equal to those of the pressure-inlet zone. The zones representing the walls are considered as stationary walls. The inlet pressure boundary has been used to apply both the inlet air and water conditions. A uniformly distributed two phase flow has been assumed to represent the experimental use of a full cone nozzle droplet spray. Each inlet cell is set to the experimental inlet pressure and a volume fraction established to inject an appropriate amount of liquid corresponding to a test condition. This adequately sets the phase inlet boundary conditions to those in practice but does not directly correspond to the test conditions where a liquid inlet mass flow combined with an air pressure inlet would have intuitively been more representative. Unfortunately, this was found not to give physically correct results and is believed to be a code related issue. With regards to the solution strategy, the solution method used was the steady-state pressure-based solver for segregated iterative procedure of the popular phase coupled SIMPLE method to resolve the pressure-velocity coupling in the flow. A Green-Gauss cell based spatial discretization for the gradient was implemented and a first order upwind spatial discretization for the density, momentum, volume fraction, turbulent kinetic energy, turbulent dissipation rate and energy gradient terms. In addition, the solution is initialized with a zero operation condition by a hybrid initialization method to obtain accurate results, then being controlled by lowering the under-relaxation factors. The energy equation was not solved for the first 1000 iterations, to ensure initial stability.

\subsection{Droplet size modelling}

The droplets are injected via a spray nozzle that produced a full cone spray at a $30^{\circ}$ spray angle and droplet diameters of the order of $400 \mu \mathrm{m}$. The spray was injected into the air flow at the entrance of a converging nozzle with an area contraction ratio of 70 and under typical operating conditions would lead to the acceleration of the air flow from a negligible Mach number to one of 0.6 , with velocities reaching $200 \mathrm{~m} / \mathrm{s}$. In doing so, we have assumed that injected droplets undergo a series of aerodynamic break up processes which result in a critical Weber number being reached. Kolev, 2007 reviews much of the current work in this topic and indicates that the critical Weber number determined from experiments generally fall in the range 5-20. The liquid injected into the converging section could produce relative velocities in the range $50-150 \mathrm{~m} / \mathrm{s}$ resulting in predicted droplet diameters of the order of 5-40 $\mu \mathrm{m}$. There is some evidence that this range is consistent with the limited experimental evidence of droplet sizes in sonic nozzles. Lemonnier and Camelo-Cavalcanti, 1993 measured droplet sizes at the exit of a converging nozzle for air water flow conditions and showed that sauter mean droplet diameters were in the range of 20-40 $\mu \mathrm{m}$. Similar studies with steam flow by Alger, 1978 have measured droplet sizes in the range 1-10 $\mu \mathrm{m}$ but these sizes may have resulted from thermal break up processes in addition to aerodynamic. To account for this uncertainty we have assumed a single diameter droplet size (ie mono dispersed) at the inlet of the valve and obtained multiple solutions for a range of droplet diameters. For the reference 
case we have assumed a $10 \mu \mathrm{m}$ diameter and investigated the effect of droplet sizes between 5 and $35 \mu \mathrm{m}$. It was found that $1 \mu \mathrm{m}$ would match homogenous conditions.

\section{Results}

Figure 5 compares both the mixture and two fluid model predictions against the experimental data. The models assume a droplet size of 10 microns is applicable. Figure 5 indicates that both mixture and two fluid approaches both predict the general trends correctly. The mixture model correctly predicts the mass flows within $5 \%$ for liquid mass fractions less than 0.4 but for greater mass fractions errors can be as high as $15 \%$ and suggest that the non-equilibrium effects are important and need to be accounted for. The predictions of the two fluid model can be seen to be satisfactory over the range of liquid mass fractions examined and generally predict the air mass flow rate within $5 \%$.

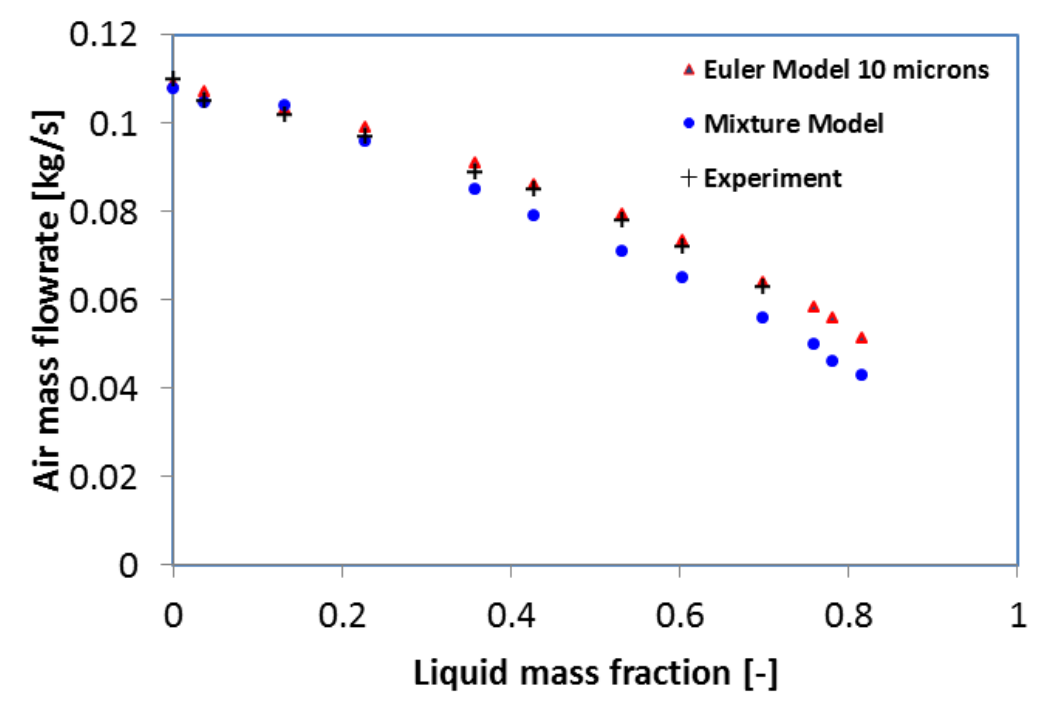

Figure 5 Experimental data and CFD predictions for an inlet of pressure 13.7 barg

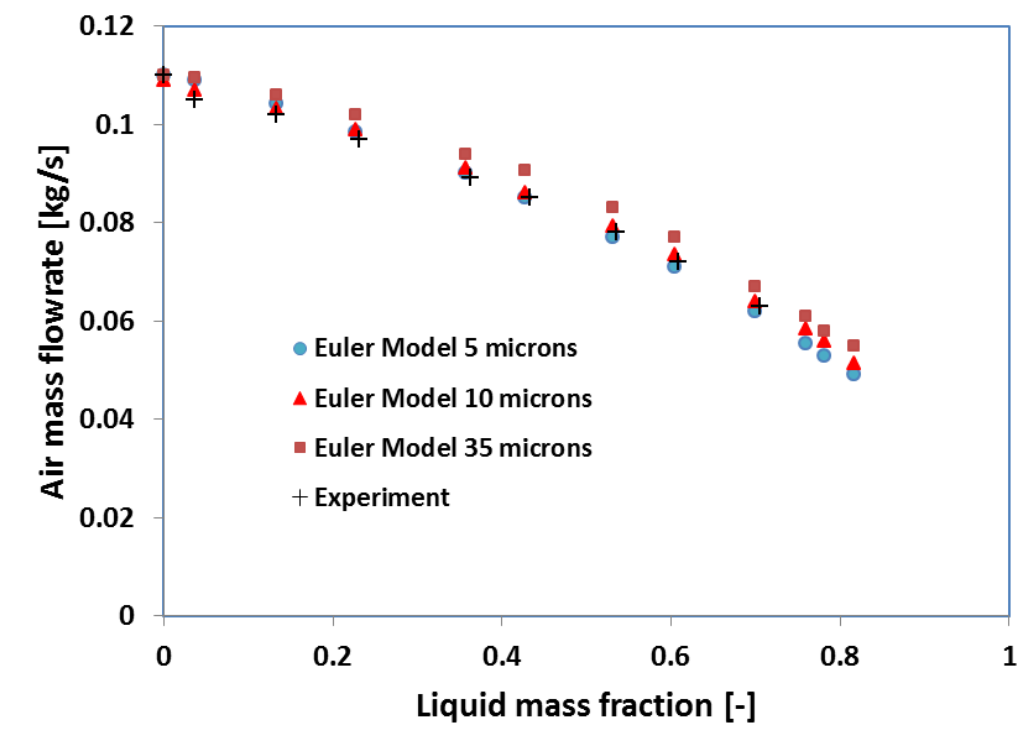

Figure 6 Experimental data and Euler-Euler predictions for droplet sizes 5-35 microns,13.7 barg 
Noting that the assumption here is for a mono dispersed droplet flow, the droplet size is one of the most uncertain parameters in the application of the model. Therefore, predictions for a range of droplet sizes have been carried out and are shown on Figure 6. This figure shows the effect of using droplet sizes from 5-35 microns and indicates an increasing influence as the liquid fraction increases, leading to an increase in the air flowrate as the droplet size is increased. However for this range of droplet sizes the predictions of air flowrates vary only by a maximum of $8 \%$. The experimental values for the air flowrates fall within the range of flowrates. Thus for an estimated droplet size based on a critical weber number of $\mathrm{We}=12$ reasonable predictions of mass flow can be achieved.
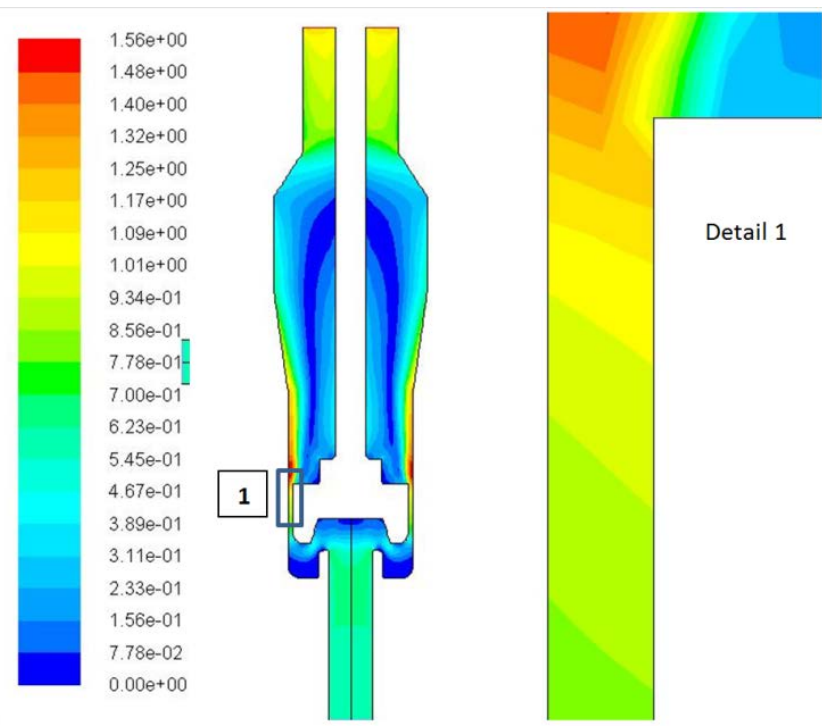

Figure 7: Two fluid model Mach Number at 13\% liquid mass fraction

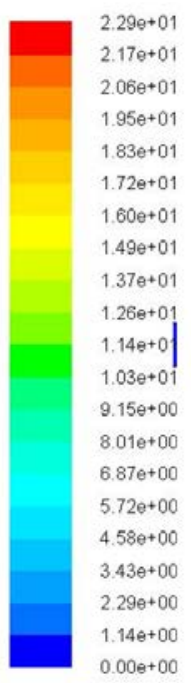

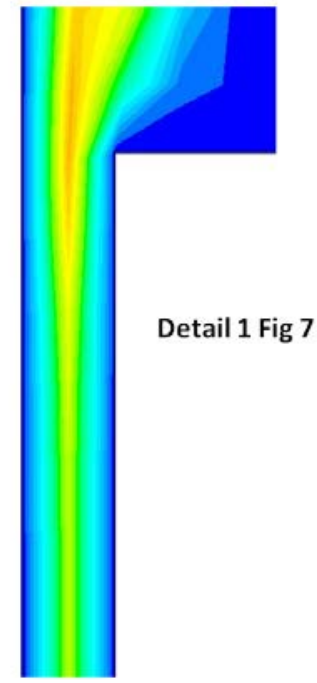

Liquid mass fraction of $13 \%$
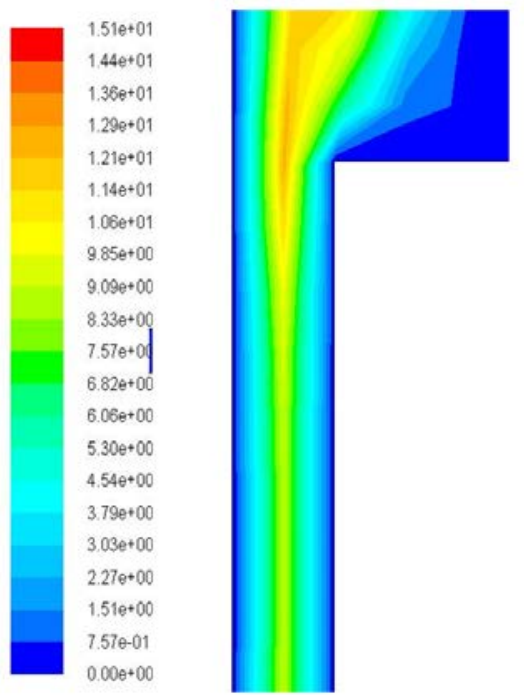

Liquid mass fraction of $70 \%$

Figure 8: Two fluid model Weber number at $13 \%$ and $70 \%$ liquid mass fraction

Further verification can be achieved by examining the predicted Weber numbers. Figure 7 shows the predictions of Mach number from the Euler-Euler model at a liquid mass fraction of $13 \%$ and indicate that the critical flow location occurs at the end of the channel between the piston and valve body. This is the choking location for all flow conditions. Figure 8 shows the 
predicted Weber numbers in this location for the 10 micron diameter case and for liquid fractions of $13 \%$ and $70 \%$.The figure shows that Weber numbers generally lie in the $10-20$ range confirming the initial assumptions and the droplet diameters investigated

\section{CONCLUSIONS}

A CFD based study has been carried out to examine the capability of predicting critical flows in a safety valve exposed to a two component (air/water) two phase flow. The results indicate that;

(i) Established two fluid modelling approaches as exemplified by the FLUENT two fluid model with standard closure relations can satisfactorily predict the two phase critical flowrates.

(ii) Droplet size modelling can be adequately addressed using a critical Weber number approach. A critical Weber number of 12 and modelling the droplet conditions as a mono dispersed flow has been shown to produce self-consistent results.

\section{REFERENCES}

1. Alger T.W. Droplet phase characteristic in liquid-dominated steam-water nozzle flow, PhD Thesis, Lawrence Livermore Laboratory, University of California, 1978

2. Darby R. On two-phase frozen and flashing flows in safety relief values: Recommended calculation method and the proper use of the discharge coefficient. Journal of Loss Prevention in the Process Industries, July 2004

3. Dempster W.M., Lee C. K., and Deans J., Prediction of the flow and force characteristics of safety relief valves, Proceedings of PVP2006-ICPVT-11 2006 ASME Pressure Vessels and Piping Division Conference, July

4. Dempster W and Elmayyah W. Two phase discharge flow prediction in safety valves. International Journal of Pressure Vessels and Piping, 110. pp. 61-65. ISSN 03080161, 2013

5. Elmayyah W. Theoretical and experimental investigations of multiphase flow in safety relief valves $\mathrm{PhD}$ Thesis, Department of Mechanical and Aerospace Engineering, University of Strathclyde Glasgow, May 2010.

6. Fluent 6.3 User Guide, 2006

7. ISO standard, Safety devices for protection against excessive pressure Part 10: Sizing of safety valves for gas/liquid two-phase flow, ISO-4126-10, 2010

8. Kolev N I, Multiphase Flow Dynamics 2 Thermal and Mechanical Interactions, $3^{\text {rd }}$ edition , Springer 2007, isbn 978-3-540-69834

9. Lemonnier H, Camelo-Cavalcanti E.S., Droplet size and velocity at the exit of a nozzle with two component near critical and critical flow, National Heat Transfer Conference; Atlanta, GA (United States); 8-11 Aug 1993 
10. Leung, J.C. A generalized correlation for one-component homogeneous equilibrium flashing choked flow. AIChE Journal, 1986

11. Manninen M, Taavassalo V, On the mixture model for multiphase flow, VTT publications, 1996

12. Ranz W.E. and Marshall, W. R., Vaporation from Drops, Part I,. Chem. Eng. Prog.. 48(3). 141-146. March 1952

13. Schiller L and Naumann Z. , Z. Ver. Deutsch. Ing.. 77. 318. 1935

14. Schmidt J and Egan S. Case studies of sizing pressure relief valves for two-phase flow. Chemical Engineering and Technology, 2009.

15. Song A, Cui L, Cao M, Cao W, Park Y, Dempster W, A CFD analysis of the dynamics of a direct-operated safety relief valve mounted on a pressure vessel, Energy Conversion and Management, 81 (2014) 407-419 